S. Mühlig'
H. Rinne ${ }^{1}$
F. Mehren ${ }^{1}$
F. Petermann ${ }^{1}$
U. Butt ${ }^{2}$
H. Worth

\section{Zur Praxis der stationären Asthmaschulung in der Bundesrepublik Deutschland - eine bundesweite Bestandsaufnahme}

In-patient Asthma Education and Self-Management Training in Clinical Practice a National Survey in Germany

\section{Zusammenfassung}

Hintergrund: Patientenschulungsmaßnahmen bilden nach den internationalen Therapierichtlinien einen integralen Bestandteil des modernen Asthma-Managements und stellen insbesondere in der medizinischen Rehabilitation eine Schlüsselkomponente für den langfristigen Therapieerfolg dar. Auf Basis der wachsenden empirischen Evidenz zur Effektivität von Asthmaschulungen wurden standardisierte Programme sowie Empfehlungen zur Qualitätssicherung der Schulungsangebote in der Versorgungspraxis entwickelt.

Ziel: Da bislang kaum empirische Daten zur Umsetzung standardisierter Programme in der Anwendungspraxis vorliegen, wurde diesbezüglich eine bundesweite Befragung unter stationären pneumologischen Einrichtungen in Deutschland durchgeführt.

Methoden: Auf Grundlage einschlägiger Adressenregister wurden alle stationären pneumologischen Einrichtungen kontaktiert und diejenigen Kliniken identifiziert, die regelmäßige Schulungen für Asthmatiker durchführten. 65 teilnehmende Kliniken (Drop-out-Quote: 12\%) wurden mittels Fragebogen ausführlich zu inhaltlichen und formalen Aspekten ihrer Schulungspraxis befragt.

Resultate: Obwohl $91 \%$ der Kliniken angaben, sich an die Expertenempfehlungen zu halten, betrug der Anteil standardisierter und evaluierter Schulungsprogramme an allen bestehenden Angeboten lediglich $50 \%$, und sogar nur in $19 \%$ der Fälle wurden diese entsprechend der jeweiligen Programminstruktionen praktiziert (Treatment-Integrität). Die konkrete Durchführung der Schulung in den einzelnen Kliniken wies so große Divergenzen auf, dass kaum von einem einheitlichen Schulungsstandard in der stationären Versorgung gesprochen werden kann.

\section{Abstract}

Background: According to international guidelines patient education is a key component of modern asthma-management. Especially in rehabilitation, patient training is considered essential for long-term treatment success. Based on growing empirical evidence for clinical efficacy of asthma self-management training, standardized education programs as well as guidelines and recommendations for quality management in health care practice have been developed.

Aim: Due to the lack of evidence [empirical data] on compliance to these recommendations we conducted a nation-wide survey including in-patient pneumological clinics in Germany.

Methods: Exploiting address registers of national asthma organizations (i.e. Deutsche Atemwegsliga) we identified 75 clinics that offer education programs on a regular basis, 65 of which participated in this study (drop-out-rate: 12\%). These institutions were asked to complete a questionnaire in order to assess various aspects of their education practice.

Results: Although $91 \%$ of clinics reported to adhere to guidelines and recommendations for patient education, merely $50 \%$ supplied standardized and evaluated education and self-management training programs. A rather small proportion (19\%) was found to fully adhere to protocol (maximum treatment integrity, no mixing of different education schemes). Furthermore, substantial variance between clinics was observed in actual performance of asthma education, the major indicators being: indication criteria and eligibility of patients; education contents; group size, duration and frequency of sessions; extent and intensity; didactical methods; number and qualification of trainers; measures of quality management, and settings. Accordingly, a

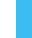


Schlussfolgerungen: Während die Patientenschulung in der stationären pneumologischen Rehabilitation heute einen hohen Versorgungsgrad besitzt, sind trotz großer Fortschritte in den letzten Jahren noch Defizite hinsichtlich der Umsetzung der Qualitätsstandards für ein effizientes Patiententraining zu konstatieren. consistent standard of patient education in rehabilitation practice has not yet been achieved.

Conclusion: While substantial advances in the proposition of patient education and self-management trainings in in-patient health care remain unquestioned, there are some deficits in actually performing such programs in clinical practice. Results also show that another question needs to be addressed: qualifying trainers. Less than half of trainers participating in this study reported to have absolved a train-the-trainer-course.

\section{Einleitung}

Die Schulung der Patienten bildet nach den nationalen und internationalen Therapierichtlinien heute einen integralen Bestandteil des modernen Asthma-Managements [1 -3] und stellt insbesondere in der medizinischen Rehabilitation eine Schlüsselkomponente für den langfristigen Therapieerfolg dar [4]. „Patientenschulung“ lässt sich allgemein definieren als eine systematische Informations- und Kompetenzvermittlung mit umschriebenen Zielen, spezifischen Inhalten und standardisierten Methoden, die eine Veränderung im Wissen, der Einstellung und im Verhalten von Patienten erzielen und ihren eigenverantwortlichen Umgang mit der Erkrankung stärken soll [5]. Das moderne stationäre Patiententraining legt den Schwerpunkt verstärkt auf die verhaltenspsychologische Komponente und integriert dementsprechend systematisch Wissens- und Kompetenzvermittlung, Verhaltens- und Fertigkeitstraining (praktisches Üben) sowie körperliches Training wie Sporttherapie oder Atemgymnastik [6,7]. Langfristig sollen durch derartige Schulungs- und Trainingsmaßnahmen die körperliche Leistungsfähigkeit der Patienten erhalten, Exazerbationen und eine Prognoseverschlechterung verhindert, die subjektive Lebensqualität verbessert und eine Kosteneinsparung für das Gesundheitswesen erzielt werden [8].

Mit wachsender empirischer Evidenz ist festzustellen, dass Patientenschulungs- und Trainingsprogramme bei Asthmatikern die Ergebnisse der medizinischen Therapie statistisch und klinisch signifikant verbessern können. Mittlerweile liegen mehrere Metaanalysen und systematische Reviews zur Effektivität von Patientenschulungs- und -trainingsprogrammen bei Asthma vor. Devine [9] fand in einer umfangreichen Meta-Analyse (31 Evaluationsstudien von 1972 bis 1993) u.a. statistisch signifikante Verbesserungen bezüglich der Zahl der Asthmaanfälle, einiger Lungenfunktionsparameter $\left(\mathrm{FEV}_{1}, \mathrm{FEV}_{1} / \mathrm{VC}\right.$, Peak-Flow), der Medikamenten-Compliance, der Lebensqualität, außerplanmäßiger Inanspruchnahme ärztlicher Hilfe, dem Bedarf an bronchialerweiternden Medikamenten sowie den Fertigkeiten der Arzneimittelanwendung (Inhalationstechnik). Clark und Nothwehr [10] ermittelten in einem systematischen Review über 18 Evaluationsstudien (1979-1995; MEDLINE, EMBASE) fünf Kategorien, in denen signifikante Verbesserungen erzielt wurden: 1) Asthmawissen, 2) Krankheitswahrnehmung und psychische Befindlichkeit, 3) Medikamentengebrauch, Inhaleranwendung und Triggervermeidung, 4) körperliche Funktionsfähigkeit und Symptomkontrolle, 5) Inanspruchnahmeverhalten medizinischer Dienstleistungen.
Insbesondere in einer Reihe von neueren prospektiven und randomisierten Kontrollgruppenstudien wurde die Effektivität von Patientenschulungs- und -trainingsprogrammen für erwachsene Asthmatiker bezüglich unterschiedlicher klinischer Outcomes überzeugend nachgewiesen [11-29]. Diese Befundlage wird durch einen systematischen Review der Cochrane Collaboration über 25 randomisierte Kontrollgruppenstudien zur Evaluation von Asthmapatientenschulungen (auf Grundlage des Cochrane Airways Group Trials Register) eindrucksvoll bestätigt. Gibson, Coughlan, Wilson, Abramson, Bauman, Hensley und Walters [30] ermittelten, dass Trainingsprogramme zum Selbstmanagement bei erwachsenen Asthmatikern

- Hospitalisierungen (OR: 0,57, 95\% CI, 0,38-0,88),

- Notfallaufnahmen (OR: 0,71, 95\% CI, 0,57-0,90),

- außerplanmäßige Arztbesuche (OR: 0,57, 95\% CI, 0,40-0,82),

- Schulfehl- oder AU-Tage (OR: 0,55, 95\% CI, 0,38-0,79) und

- nächtliche Asthmabeschwerden (OR: 0,53, 95\% Cl, 0,39-0,72)

signifikant reduzieren, während die Lungenfunktion kaum beeinflusst wird. Allerdings weisen Patienten, die ihr Asthma mit Hilfe eines individualisierten Therapieschemas durch eigenständige Anpassung der Medikation selbst kontrollieren, eine bessere Lungenfunktion auf als Patienten, die ihre Medikation vom Arzt steuern lassen. Patiententrainings unter Einbeziehung eines schriftlichen Aktionsplanes zeigen durchschnittlich eine höhere Effektivität als Schulungen ohne derartige Unterlagen (OR: 0,35, $95 \% \mathrm{CI}, 0,18-0,68)$. Zusammenfassend stellen die Autoren fest, dass verhaltensnahe Patiententrainings in Kombination mit einem regelmäßigen Selbstmonitoring (entweder Peak-Flow-Metrie oder Symptombeobachtung), ärztlicher Überwachung und einem schriftlichen Aktionsplan die Therapieergebnisse deutlich verbessern. Dies gilt interessanterweise allerdings nicht für rein edukative Schulungsinterventionen: In einem zweiten analog durchgeführten Review selektieren Gibson, Coughlan, Wilson, Hensley, Abramson, Bauman und Walters [31] alle kontrollierten randomisierten Evaluationsstudien zur Asthmatikerschulung, die sich ausschließlich auf Informationsvermittlung beschränkten. Diese begrenzten Schulungsmaßnahmen führen weder zu einer Reduzierung der Hospitalisierungen, Arztkontakte und Einschränkung von Alltagsaktivitäten noch zu einer Verbesserung der Lungenfunktion oder Anwendung von Arzneimitteln (AM), sondern lediglich zu einer positiven Veränderung in der Wahrnehmung der Asthmasymptome (OR: 0,40, 95\% CI, $0,18-0,86)$. Somit ist davon auszugehen, dass rein informationsvermittelnde Schulungen keinen bedeutsamen Effekt auf den Therapieerfolg besitzen. 
Tab.1 Einsatz standardisierter und evaluierter Schulungsprogramme

\footnotetext{
- Schulungsumfang: mind. 10-15 Schulungsstunden

- optimale Gruppengröße: 8-10 Patienten

- interdisziplinäres Schulungsteam (medizinische, physiotherapeutische, psychologische und pädagogische Kompetenz)

- Koordination zwischen Schulern (inhaltl. Abstimmung u. klare Arbeitsteilung)

- Train-the-Trainer-Ausbildung der Schuler und vorangegangene Hospitation bei erfahrenen Kollegen (Schulerqualifikation)

- differenzielle Indikation für Patientenschulung (diagnosespezifische Zielgruppe, Indikation nach Motivation, Schulbarkeit und Schulungsbedarf)

- patientenorientierte Methodik-Didaktik (weniger Vortragsstil, mehr Dialog und Übungen)

- Einsatz von multimedialen Schulungsmaterialien (Demonstrationsobjekte, AM-Systeme, Peak-Flow-Geräte, Flip-Charts, Schautafeln, Bilder, Videos etc.)

- geeignete Räumlichkeiten und Ausstattung (Platz f. ca. 20 Personen, angenehme Atmosphäre, gute Sichtverhältnisse und Akustik, apparative Ausstattung)

- Verstetigung des Schulungseffektes (Erfolgskontrolle, Einbeziehung der Lebenspartner, Nachschulungsangebote; Aushändigung eines schriftlichen Aktionsplanes)
}

Um die potenzielle Effektivität von Patiententrainings auch in der Versorgungspraxis zu gewährleisten, wurden verschiedentlich Kriterien zur Qualitätssicherung der Asthma-Patientenschulung diskutiert und erste evidenzbasierte Empfehlungen entwickelt, die in Tab. 1 zusammengefasst sind [8,32].

Patiententrainings können auf Dauer dann am effizientesten realisiert werden, wenn sie sich auf standardisierte und wissenschaftlich geprüfte Programme stützen [33]. Die Standardisierung von Schulungsprogrammen bildet dabei eine unabdingbare Voraussetzung für eine dauerhafte Qualitätssicherung, indem sie gewährleistet,

- dass sie beliebigen Schulern unabhängig von deren Vorwissen und Qualifikation in systematischer Weise vermittelt (Lehrbarkeit in „Train-the-Trainer“-Kursen),

- von diesen in homogener Weise umgesetzt (Reproduzierbarkeit),

- mit zumutbarem Aufwand in anderen Kliniken implementiert (Praktikabilität und Anwendungsökonomie),

- klinikintern auf ihre Qualität überprüft und klinikübergreifend wissenschaftlich evaluiert werden können (Überprüfbarkeit) und

- dass Patienten damit in unterschiedlichen Einrichtungen vergleichbare Angebote auf gesichertem Niveau erhalten (Replizierbarkeit/Vergleichbarkeit).

Zudem beziehen sich die beeindruckenden empirischen Nachweise der Wirksamkeit, Unbedenklichkeit, Praktikabilität und Effizienz von Patiententrainings natürlich nur auf die jeweils geprüften Programme und besitzen nur dann Gültigkeit, wenn diese auch in der standardisierten Form durchgeführt werden $[34,35]$. In Deutschland stehen bislang im Wesentlichen zwei standardisierte und ausreichend evaluierte Schulungsprogramme für die stationäre Asthmaschulung zur Verfügung: das Bad Reichenhaller Modell $[7,36,37]$ und das in Düsseldorf entwickelte Schulungsprogramm „Asthmabehandlungs- und Schulungssystem (ABUS)“ “ $[14,38-42]$. Während heute in praktisch allen Rehabilitationskliniken für Atemwegserkrankungen sowie in zahlreichen pneumologischen Fachabteilungen von Akutkrankenhäusern und Facharztpraxen regelmäßige Patientenschulungen stattfinden [43-45], stellt sich die Frage, inwieweit in der Praxis dabei die standardisierten und evaluierten Schulungsund Trainingsprogramme eingesetzt bzw. adäquat angewendet werden (Treatment-Integrität).

Zur Klärung der Versorgungsrealität der stationären Asthmaschulung wurde vom Zentrum für Klinische Psychologie und Rehabilitation der Universität Bremen in Kooperation mit der Deutschen Atemwegsliga eine bundesweite Befragung unter stationären Einrichtungen durchgeführt, die überwiegend oder in signifikantem Ausmaß Patienten mit chronisch-obstruktiven Atemwegserkrankungen behandeln.

\section{Methoden}

Auf Grundlage der Adressenkartei der Deutschen Atemwegsliga, der aktualisierten Fassung des Patientenschulungsführers „Wer bietet was?" [44] sowie ergänzender Recherchen in Adressenregistern und im Internet wurden insgesamt 114 stationäre pneumologische Einrichtungen bzw. Abteilungen identifiziert, in denen potenziell Asthmaschulungen für erwachsene Patienten angeboten wurden. Diese wurden von April bis August 2000 angeschrieben (Nachbefragungen bis September 2001) und mittels strukturiertem Fragebogen zu ihrer Patientenschulungspraxis befragt. Von den ermittelten Einrichtungen waren zwischenzeitlich 13 geschlossen worden, 22 hatten die Indikation gewechselt oder waren nicht primär auf erwachsene Patienten mit chronisch-obstruktiven Atemwegserkrankungen ausgerichtet. In weiteren fünf Kliniken oder Klinikabteilungen war der Anteil der Asthmatiker an der behandelten Patientenpopulation so weit abgesunken, dass inzwischen keine speziellen Schulungsangebote mehr durchgeführt wurden. Somit verblieben insgesamt 74 stationäre Einrichtungen (72 Einrichtungen der medizinischen Rehabilitation und zwei Akutkliniken), in denen in signifikantem Umfang erwachsene Asthmapatienten geschult werden. Da nach wiederholter telefonischer Nachfrage insgesamt sieben Kliniken den Fragebogen nicht beantworteten und nur zwei Kliniken die Teilnahme an der Befragung verweigerten (Drop-out-Rate: 12\%), beziehen sich die im Folgenden dargestellten Resultate auf eine Umfrage unter 65 stationären pneumologischen Einrichtungen (34\% LVA; 23\% BfA; 45\% Privat; $35 \%$ andere Träger).

\section{Ergebnisse}

In $92 \%$ der schulenden Kliniken bildet die Patientenschulung für Asthmatiker einen integralen Bestandteil im Behandlungsplan, der in $88 \%$ der Fälle in festen Terminblöcken angeboten wird, wobei die Teilnahme in $85 \%$ der Einrichtungen für die Patienten sogar obligatorisch ist. Die Entscheidung über die Schulungsteilnahme wird in $99 \%$ vom Arzt, in $27 \%$ vom Patienten und in $11 \%$ durch andere Personen getroffen (Mehrfachnennungen möglich). Die mittlere tatsächliche Teilnahmequote an den Schulungsangeboten (Inanspruchnahme) liegt bei $72 \%$. Zusätzliche Therapieangebote bestehen vorwiegend in Atemschulungen (95\%), Raucherentwöhnung (88\%), Asthmasport (57\%) sowie anderen Maßnahmen wie Atemtherapie, Entspannungsverfahren oder Qi-Gong (insg. 60\%). 
Obwohl $91 \%$ der befragten Klinken angeben, sich bezüglich der Schulungsdurchführung an den o.g. Expertenempfehlungen zu orientieren, bestehen bei genauerer Betrachtung noch deutliche Defizite bei der Umsetzung dieser Qualitätsstandards. Bereits vor einigen Jahren war in einer Untersuchung von Petro [45] festgestellt worden, dass nur in weniger als $20 \%$ der damals befragten ambulanten und stationären pneumologischen Einrichtungen (Erwachsene und Kinder) evaluierte Schulungsprogramme eingesetzt wurden, während die überwiegende Mehrheit der Schuler kostenlose Materialien der Pharmaindustrie oder selbst entwickelte Schulungen benutzte, deren Effektivität wissenschaftlich nicht belegt ist. Die Resultate unserer Klinikbefragung fallen zwar wesentlich positiver aus, erreichen aber ebenfalls noch kein zufriedenstellendes Niveau. Evaluierte Schulungsprogramme („Bad Reichenhaller Modell“, „Asthmabehandlungsund Schulungsprogramm - ABUS“, „Ambulante Fürther Asthmaschulung - AFAS") werden mittlerweile in immerhin der Hälfte (50\%) der Kliniken eingesetzt (Abb.1), während die andere Hälfte der stationären Einrichtungen noch immer wissenschaftlich nicht geprüfte Schulungen durchführt (z. B. „Lebensrhythmus Atmen“, „Lernen-Wissen-Können“, „Ingelheimer Modell“, BfASchulung, Eigenentwicklungen). Unter den evaluierten Programmen besitzt das Bad Reichenhaller Modell (46\%) die größte Verbreitung, gefolgt vom ABUS ( $32 \%$ ) und AFAS (22\%), wobei letzteres allerdings für den ambulanten Bereich konzipiert und evaluiert wurde.

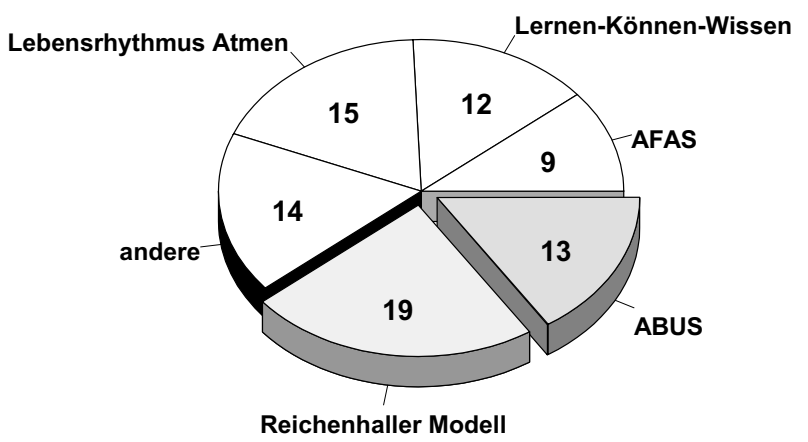

Abb. 1 Verteilung der eingesetzten Schulungsprogramme (Anzahl Nennungen, Mehrfachnennungen möglich).

Zudem werden in jeder zweiten Klinik (52\%), die sich auf standardisierte Schulungsmodelle stützt, mehrere unterschiedliche Programme in Kombination verwendet und damit die Treatment-Integrität der evaluierten Schulungen verletzt. In immerhin fast jeder vierten Klinik (23\%) existiert nicht einmal ein schriftlich fixiertes Schulungskonzept, so dass hier nicht von einem standardisierten Vorgehen auszugehen ist. Dementsprechend findet sich in der Praxis der stationären Asthmaschulung eine divergierende Auswahl und Zusammenstellung der Schulungsinhalte, die sich gelegentlich eher an den persönlichen Präferenzen oder Fähigkeiten des Schulers als am Schulungskonzept zu orientieren scheint. Im Durchschnitt ist aber eine relativ hohe Übereinstimmungsquote der Schulungsinhalte in den verschiedenen Einrichtungen festzustellen, wobei die medizinischen Themenbereiche deutlich verbreiteter sind als die psychologischen (Abb. 2).

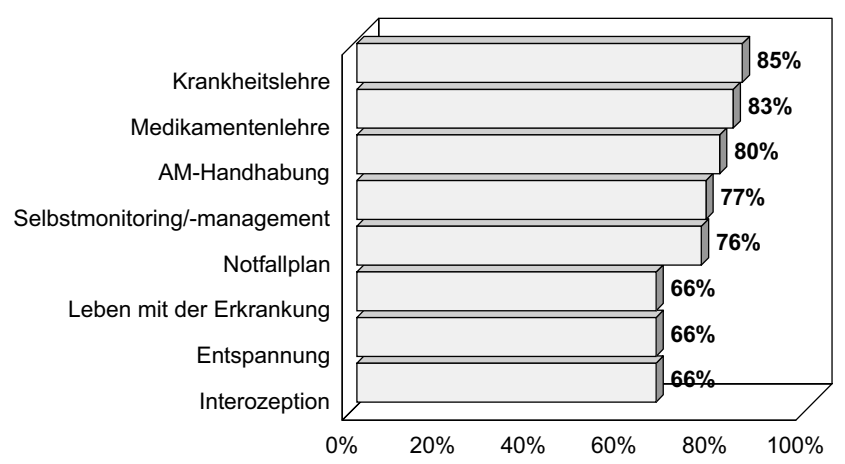

Abb. 2 Anteil der Kliniken, die bestimmte Schulungsmodule anbieten (in Prozent).

Im Gegensatz zu den Intentionen der Programmentwickler und den Expertenempfehlungen ist in der Schulungspraxis eine mangelnde Indikationsgruppenspezifität der „Asthma-Schulungen“ zu konstatieren. In nur der Hälfte der befragten Kliniken (51\%) bildet die Diagnose das primäre Zuweisungskriterium für eine Schulungsmaßnahme (Abb.3); weitere Entscheidungskriterien für die Schulungsteilnahme sind die Motivation (52\%), das kognitive Niveau (46\%), die Deutschsprachigkeit (37\%) und das Alter der Patienten (28\%). Nur in jeder vierten Klinik (26\%) existieren diagnosespezifische Schulungsgruppen, so dass mehrheitlich Patienten mit unterschiedlichen Atemwegserkrankungen (z.B. Asthma, COPD, Emphysem) gemeinsam geschult werden.

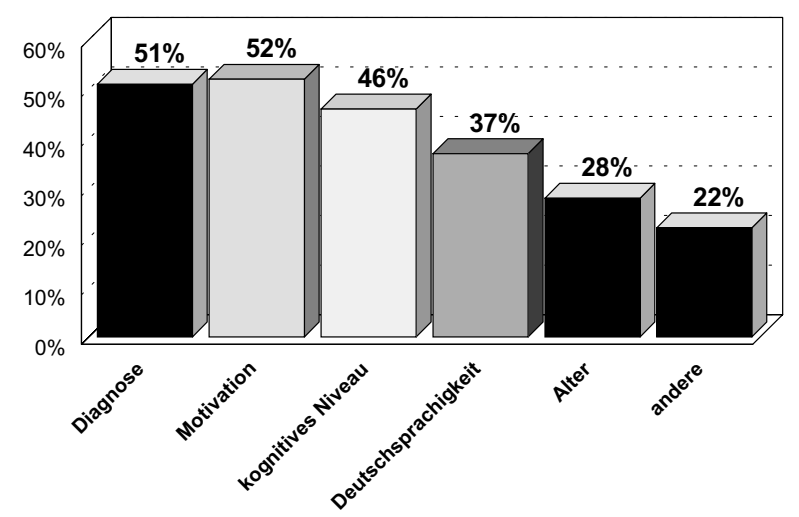

Abb. 3 Zuweisungskriterien für Schulungsmaßnahme (in Prozent).

Eine besonders ausgeprägte Heterogenität ist hinsichtlich der praktischen Schulungsdurchführung in unterschiedlichen Kliniken festzustellen. So variiert die Größe der Schulungsgruppen zwischen den Einrichtungen absolut von drei bis zu mehr als 60 Teilnehmern. Da die Teilnehmeranzahl für die verschiedenen Schulungskomponenten eine unterschiedliche Bedeutung besitzt, wurde die durchschnittliche Minimal- und Maximalteilnehmerzahl themenspezifisch differenziert erfragt. Es zeigt sich folgende Variation der mittleren Teilnehmerquote in Bezug auf die thematischen Schulungsmodule (Abb. 4): Die höchste durchschnittliche Gruppengröße ist bei den medizinischen Themen wie „Krankheitslehre“ und „Medikamentenlehre“ zu verzeichnen, gefolgt von den Schulungsbausteinen „Interozeption“, „Leben mit der Erkrankung“, „Selbstmonitoring/Selbstmanagement“ und „Entspannungsverfahren“, wohingegen die geringste Teilnehmerzahl bei den Themen „AM-Handhabung“ und „Notfallplan" besteht. 


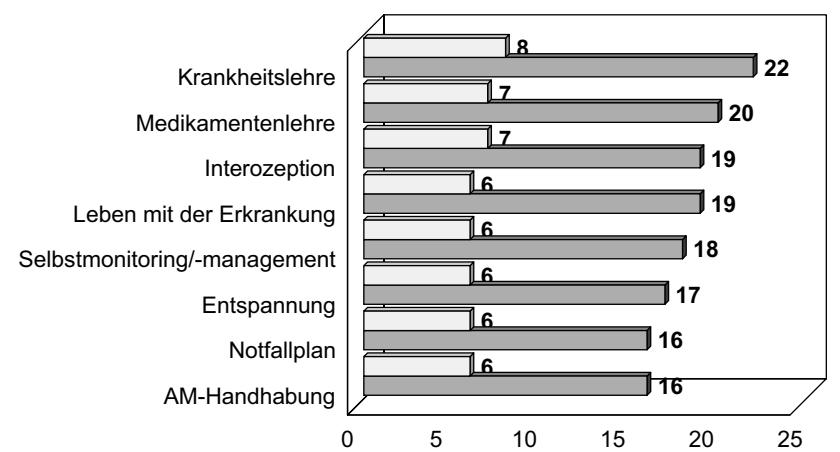

Abb. 4 Durchschnittliche Minimal- und Maximalteilnehmerzahl nach thematischen Modulen.

Auch die Anzahl (eine bis mehr als acht Doppelstunden), Dauer (ca. 30-120 Minuten) und Frequenz der Schulungseinheiten (täglich bis 1-mal in drei Wochen) variiert erheblich, so dass von einer wenig vergleichbaren Schulungsintensität in unterschiedlichen Einrichtungen der stationären Versorgung ausgegangen werden muss. Der durchschnittliche Schulungsumfang beträgt 9 Schulungsstunden ( $=546 \mathrm{Min}$.), wobei auch hier eine extreme Schwankungsbreite (min.- max.: 1-34 Std. ${ }^{1}$ ) besteht. Die Schulungsstunden verteilen sich dabei folgendermaßen auf die einzelnen thematischen Module (Abb.5): Den mit Abstand größten Umfang nimmt überraschenderweise das „Entspannungstraining“ (139 Min.) ein, gefolgt von der "Krankheitslehre“ (92 Min.), „Medikamentenlehre“ (90 Min.) und „Selbstmanagement“ (74 Min.), während der Schulungsumfang für „AM-Handhabung“ (67 Min.), „Leben mit der Erkrankung“ (65 Min.), ,Interozeptionstraining“ (55 Min.) und „Notfallplan“ (47 Min.) deutlich geringer ausfällt.

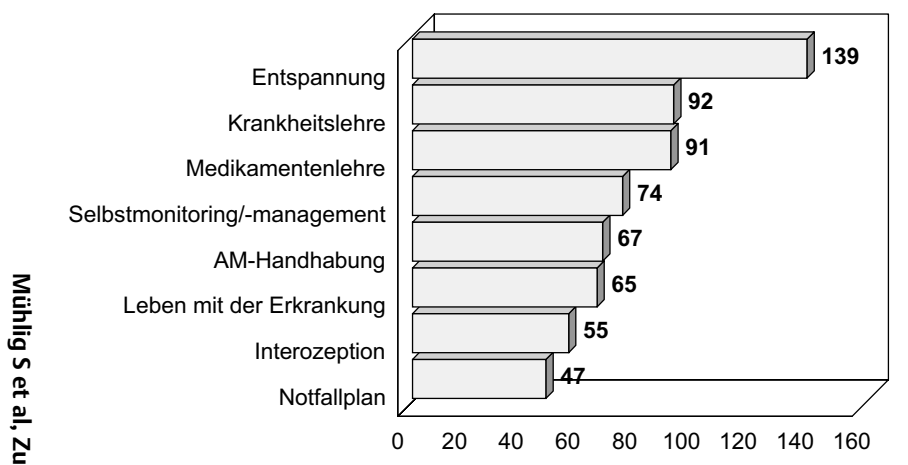

Abb. 5 Durchschnittlicher Schulungsaufwand nach thematischen Modulen (in Min.).

Bezüglich des konkreten methodisch-didaktischen Vorgehens stellt in der stationären Patientenschulung der edukative Frontalunterricht bzw. Vortragsstil (32\%) gegenüber den von Expertenseite empfohlenen multimedialen und übenden Vermittlungsformen „Verhaltensübung“ (25\%), „Demonstrationen“ (22\%) oder „Dialog“ (17\%) die häufigste Variante dar (Abb. 6). Allerdings wird die rein edukativ-informationsvermittelnde Form der Patientenschulung insgesamt nur noch in knapp einem Drittel der Fälle praktiziert, während sich überwiegend das Patiententraining i.e.S. durchgesetzt hat.

${ }^{1}$ sowie ein „Ausreißer“ mit 61 Std.

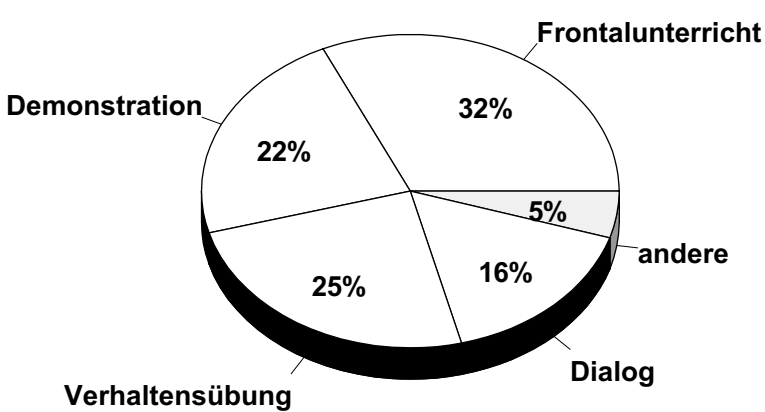

Abb. 6 Methodisch-didaktisches Vorgehen (Selbstangaben).

Der Einsatz von Medien stellt sich dabei folgendermaßen dar (Abb.7): Es dominiert die Darstellung mittels Overheadfolien (20\%), Flipchart (16\%), Demonstrationsmodellen (14\%) oder Broschüren (13\%), während elektronische Medien (Dias: $11 \%$; Videos: $9 \%$ ) sowie überraschenderweise auch Medikamentenmuster $(5 \%)$ und Hilfsmittel (Peak-Flow-Geräte: $5 \%$ ) seltener zum Einsatz kommen.

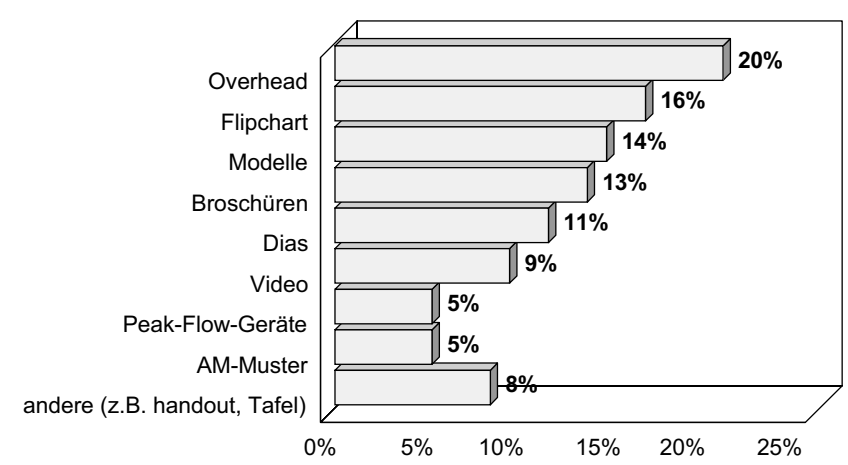

Abb. 7 Verteilung des Einsatzes unterschiedlicher Schulungsmedien.

Erhebliche Defizite sind demgegenüber in Bezug auf Maßnahmen zur Qualitätssicherung und Verstetigung des Schulungseffektes festzustellen (Abb. 8): So werden den Patienten zwar in $71 \%$ der Kliniken Nachschulungen empfohlen, aber nur in Ausnahmefällen Auffrischungssitzungen (booster-sessions) auch selbst angeboten (16\%). Demgegenüber werden immerhin in $53 \%$ der Fälle die Lebenspartner einbezogen und erfreulicherweise sogar in $65 \%$ schriftliche Zusammenfassungen der Schu-

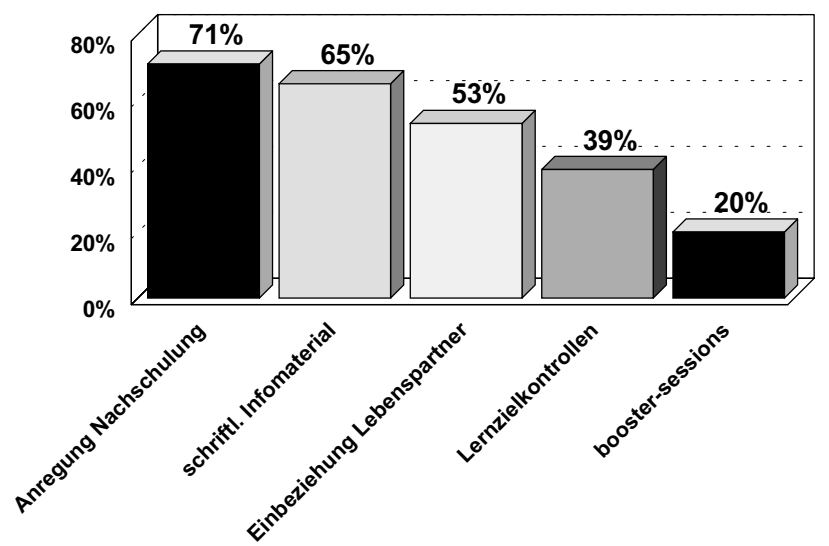

Abb. 8 Maßnahmen zur Verstetigung der Schulungseffekte. 
lungsinhalte ausgehändigt. Qualitätssicherungsmaßnahmen wie Erfolgskontrollen der Schulung (z.B. mittels Wissensfragebogen) werden nur in einer Minderheit der Kliniken (39\%) durchgeführt, so dass festgestellt werden muss, dass die Schulungseffekte in den meisten Fällen ungeprüft bleiben. Auch Qualitätszirkel zur Kontrolle und Verbesserung der Schulungsangebote werden nur in einer Minderheit (20\%) der Kliniken durchgeführt.

Die Anzahl sowie die Fach- und Schulungsqualifikation, die Berufspraxis und die Trainingserfahrung der Schuler weisen ebenfalls eine erhebliche Spannbreite auf. So variiert die Anzahl der in die Patientenschulung involvierten Mitarbeiter pro Klinik zwischen 1-15 Personen. Nach Berufsgruppen differenziert zeigt sich, dass die Ärzte fast die Hälfte (46\%) der Schuler stellen, während Psychologen/Pädagogen (17\%), Pflegepersonal (15\%) und Physiotherapeuten (14\%) drei annähernd gleich große Gruppen bilden (Abb.9).

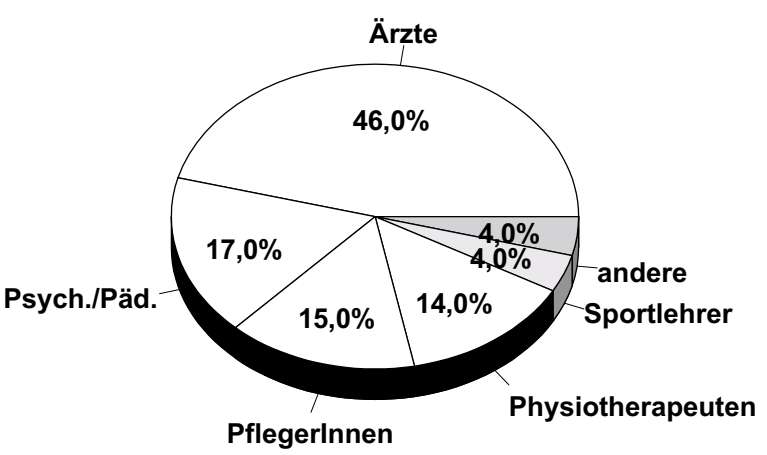

Abb. 9 Prozentuale Verteilung der Schuler $(n=360)$ nach Berufsgruppen.

Die Patientenschuler absolvieren dabei im Jahr durchschnittlich 52 Unterrichtseinheiten, wobei die Anzahl der individuellen Schulungsstunden zwischen drei und 300 variiert. Betrachtet man allerdings die individuelle Anzahl der geleisteten Schulungen pro Jahr nach Berufsgruppen, zeigt sich, dass die Physiotherapeuten die mit Abstand größte Schulungspraxis besitzen (86 Schulungen/p.a.), gefolgt von den Pflegeberufen (65) sowie den Sportlehrern (58), während Psychologen/Pädagogen (48) und Ärzte (38) deutlich weniger Schulungen pro Person leisten. Ein relativ hoher Anteil der Schulungen (49) wird von diversen anderen Berufsgruppen abgehalten (z.B. Atemtherapeuten, Bademeister usw.).

Hinsichtlich der speziellen Schulungsqualifikation ist festzustellen, dass nicht einmal jeder zweite (43\%) der in der Patientenschulung tätigen Mitarbeiter sich in einem Train-the-Trainer-Seminar fortgebildet oder bei einem berufserfahrenen Kollegen hospitiert (44\%) hat (Abb. 10). Der größte Anteil speziell trainierter Schuler findet sich mit 55\% unter den Pflegeberufen, gefolgt von den Ärzten (44\%) und Psychologen/Pädagogen (40\%), während Sportlehrer (29\%) und Physiotherapeuten (30\%) nur zu gut einem Viertel bis einem Drittel ein Trainerzertifikat besitzen. Die durchschnittliche Berufserfahrung als Patientenschuler liegt bei 6 Jahren (min. - max.: 1-22 Jahre), wobei die Ärzte im Schnitt am längsten schulen (7J.), beinahe so lange die Physiotherapeuten (6), während Pfleger (5), Psychologen/Pädagogen (5) und Sportlehrer (3) deutlich weniger Berufsjahre in schulender Tätigkeit besitzen.

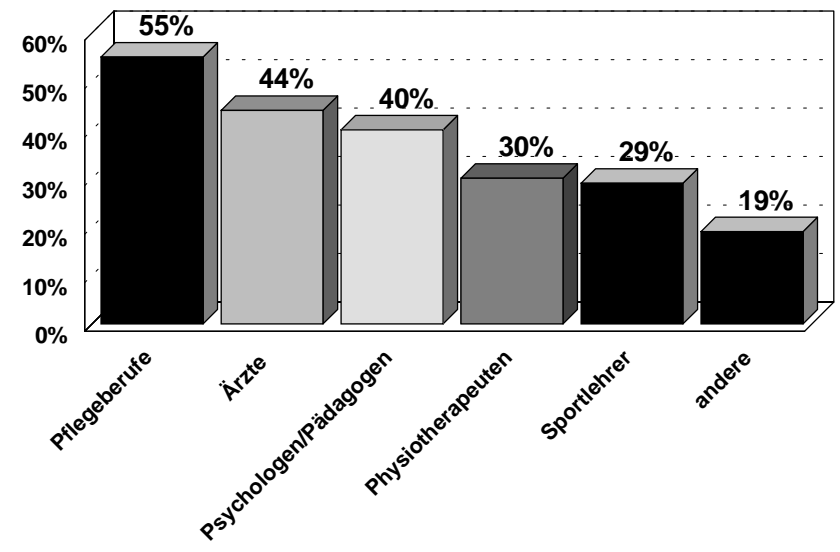

Abb. 10 Schulerqualifikation (Train-the-Trainer-Seminar) nach Berufsgruppen.

Die Kooperation innerhalb des Schulungsteams scheint in vielen Kliniken noch verbesserungsfähig. Obwohl die Schuler sich in 89\% der Kliniken inhaltlich abstimmen, finden regelmäßige Teamsitzungen der Schulungsanbieter nur in knapp der Hälfte der Fälle $(45 \%)$ statt. Demgegenüber scheint die Ausstattung der Kliniken mit Schulungsräumen und -materialien überwiegend unproblematisch: Fast alle Kliniken (95\%) besitzen spezielle Schulungsräumlichkeiten ausreichender Größe (durchschnittlich über 50 qm), mit sicheren Aufbewahrungsmöglichkeiten für Schulungsunterlagen (92\%). Die materielle Ausstattung für Schulungszwecke wird überwiegend aus Eigenmitteln (91\%), z.T. aber auch durch Sponsoren (45\%), die Klinikträger (8\%) oder andere (z. B. Pharmaindustrie, $12 \%$ ) finanziert.

\section{Diskussion}

Im Bereich der stationären pneumologischen Behandlung chronisch-obstruktiver Atemwegserkrankungen besitzt Patientenschulung heute einen hohen Versorgungsgrad und ist in nahezu allen Kliniken fest in die Gesamtbehandlung integriert. Die tatsächliche Teilnahmequote liegt bei über $72 \%$ der infrage kommenden Patienten, wobei sich auf Basis unserer Daten keine Aussagen darüber treffen lassen, inwieweit eine Nicht-Teilnahme eigenmächtig oder in Absprache mit den Behandlern erfolgte. Erfreulicherweise bieten fast alle Kliniken zusätzlich Atemschulungen und Raucherentwöhnungen an. Demgegenüber scheinen beim Asthmasport und anderen Zusatzangeboten (z. B. Entspannungsgruppen, Atemtherapie) noch Ausbaumöglichkeiten zu bestehen.

Ein offenbar schwierig zu lösendes Problem stellt die Zusammensetzung der zu schulenden Patientengruppen dar. In fast drei Viertel der Kliniken schließen die „Asthmaschulungen“ auch Patienten mit anderen Diagnosen (z.B. COPD, Emphysem) ein. Dies stellt insofern eine fragwürdige Lösung dar, als ein Großteil der Schulungsinhalte spezifisch auf Asthma bronchiale zugeschnitten ist, welche für die Teilnehmer mit anderen Diagnosen nicht nur irrelevant sind, sondern zu unnötiger Verwirrung führen können. Obwohl diese gemischten Schulungsgruppen i.d.R. den begrenzten Kapazitäten der Kliniken zuzuschreiben sind und sich argumentieren lässt, dass die Schulungsangebote auf diese Weise einer größeren Anzahl von Patienten zugute 
kommen, wären daher langfristig indikationsspezifische Gruppen anzustreben. Zudem scheinen spezielle Angebote für Patienten mit Sprachproblemen oder kognitiven Einschränkungen erforderlich, die bislang offenbar häufig nicht ausreichend erreicht werden können ${ }^{2}$. Dabei bietet sich für die stationäre Versorgung ein modularer Schulungsaufbau an, um Basisschulungsmodule für alle Patienten mit Spezialangeboten für spezifische Subgruppen effizient kombinieren zu können.

Hinsichtlich der Umsetzung der von den Fachgesellschaften definierten Qualitätsstandards und Expertenempfehlungen für ein effizientes Patiententraining sind trotz großer Fortschritte in den letzten Jahren noch einige Defizite zu konstatieren. Zunächst ist festzustellen, dass noch immer in jeder zweiten Klinik unsystematische Schulungen durchgeführt oder wissenschaftlich ungeprüfte Schulungsprogramme verwendet werden. Speziell für den stationären Bereich evaluierte Programme werden insgesamt nur in 39\% der Kliniken eingesetzt, wobei das Bad Reichenhaller Modell gegenüber dem ABUS weiter verbreitet ist. In der praktischen Schulungsdurchführung sind zudem Verletzungen der Treatment-Integrität zu beobachten, da häufig unterschiedliche Schulungsprogramme in Kombination praktiziert werden. Letztlich reduziert sich der Anteil der Kliniken, die eines der evaluierten Programme in Reinform verwenden, auf nur 19\%. Dennoch weisen die ausgewählten Schulungsinhalte eine relativ hohe Übereinstimmung zwischen den Einrichtungen auf, wobei die medizinischen Thematiken sowie die Vermittlung von Selbstmanagementkompetenzen dominieren, während die eher psychologisch orientierten Komponenten seltener angeboten werden. Die höchsten durchschnittlichen Teilnehmerzahlen sind bei den medizinischen Themen zu verzeichnen, die geringsten bei den verhaltensnahen Schulungskomponenten. Dies entspricht einer sinnvollen Verteilung der Schulungsgruppengröße, da für die praktisch übenden Schulungskomponenten kleinere Gruppengrößen angezeigt sind als für die rein informationsvermittelnden Bestandteile. Inwieweit sich in diesen Zahlen möglicherweise auch eine unterschiedliche Attraktivität für die Patienten widerspiegelt, ist auf Grundlage der Klinikangaben nicht zu entscheiden.

Die Bedingungen der Schulungsdurchführung in den einzelnen Kliniken (z. B. Gruppengröße und Schulungsintensität nach Stundenanzahl, Sitzungsdauer und -frequenz) weichen z.T. so stark voneinander ab, dass kaum von einem Schulungsstandard in der stationären Versorgung gesprochen werden kann. Es ist evident, dass derart divergierende Vorgehensweisen zu kaum vergleichbaren Schulungseffekten führen müssen. Interessanterweise sind dabei erhebliche Variationen im Schulungsumfang für die unterschiedlichen Themeninhalte festzustellen. Auffällig ist, dass Schulungsmodule, die mit relativ geringerem Vermittlungsaufwand verbunden sind („Entspannungstraining“, „Krankheitslehre“, „Medikamentenlehre“) gegenüber den Komponenten mit stärker übendem oder demonstrierendem Charakter zeitlich klar überrepräsentiert sind. Da aber gerade Letztere für ein dauerhaft erfolgreiches Selbstmanagement und eine ausreichende Patienten-Compliance von entscheidender Bedeutung sind, wäre anzu-

\footnotetext{
${ }^{2}$ Eine spezielle Schulungsversion für türkischsprachige Patienten wird derzeit von der Arbeitsgruppe Worth, Dhein und Münks-Lederer in Fürth evaluiert.
}

regen, die Schulungsangebote künftig zu deren Gunsten umzustrukturieren.

Auf Seiten der Schuler sind ebenfalls sehr unterschiedliche Bedingungen zu konstatieren. Allein die enorme Variationsbreite der Anzahl der Schuler pro Klinik (1 - 15) bedingt eine kaum vergleichbare Schulungspraxis. Insgesamt stellen die Ärzte mit 50\% die größte Schulergruppe, wohingegen Psychologen/Pädagogen, Pflegepersonal und Physiotherapeuten annähernd gleich große Minderheiten bilden. Es hat den Anschein, dass häufig eine unsystematische Zusammensetzung des Schulungsteams nach Verfügbarkeit statt Fachqualifikation vorgenommen wird. Die durchschnittliche Anzahl von Schulungsstunden pro Schuler liegt bei gut 50 Stunden jährlich (mit einer erheblichen Spannbreite von drei bis 300 Std./p.a.). Im Vergleich der jährlichen Schulungsstunden nach Berufsgruppe wird deutlich, dass die Gruppe der Physiotherapeuten mit Abstand die höchste Schulungsstundenzahl leistet, Psychologen/Pädagogen, PflegerInnen und Sportlehrer einen Mittelplatz einnehmen, während die Ärzte eine deutlich geringere individuelle Schulungspraxis aufweisen.

Hinsichtlich der speziellen Schulungsqualifikation besteht offenbar noch Nachholbedarf. Weniger als jeder zweite Schulungsanbieter besitzt eine Train-the-Trainer-Fortbildung (bzw. hat wenigstens bei einem berufserfahrenen Kollegen hospitiert), d.h. über die Hälfte der Schulungspraktiker muss diese relativ schwierige Aufgabe im Learning-by-doing-Verfahren bewältigen. Der Anteil der fortgebildeten und zertifizierten Patientenschuler liegt unter den Pflegeberufen, Ärzten und Psychologen/ Pädagogen am höchsten - und am niedrigsten ausgerechnet bei den Physiotherapeuten, die die meisten Schulungsstunden pro Person absolvieren. Letztere weisen auch neben den Ärzten die längste Berufserfahrung in der Patientenschulung auf, während Psychologen, Pflegeberufe und Sportlehrer im Durchschnitt deutlich weniger lange schulen.

Das methodisch-didaktische Vorgehen in der stationären Patientenschulung beschränkt sich jedoch nicht auf den weniger effektiven Vortragsstil bzw. „Frontalunterricht“. Dieser bildet zwar nach wie vor die häufigste Vermittlungsform, wird aber nur noch in rund einem Drittel der Kliniken vorwiegend praktiziert. In den übrigen Fällen scheint sich das Patientenverhaltenstraining zunehmend durchzusetzen. In Bezug auf den Einsatz multimodaler Medien besteht dabei aber offenbar noch Entwicklungsspielraum, insbesondere hinsichtlich der Verwendung elektronischer Präsentationsmöglichkeiten. Überraschend gering fällt vor allem die Verwendung von AM-Mustern und Selbstmonitoringinstrumenten (PEF-Geräte) zu Schulungszwecken aus, obwohl gerade diese realen Therapiegegenstände die höchste Anschaulichkeit und Praxisrelevanz besitzen und für das Selbstmanagement-Training von essenzieller Bedeutung sind. Auch die Kooperation zwischen den einzelnen Schulern scheint noch verbesserungsfähig, da nur in der Hälfte der Kliniken Teamsitzungen abgehalten werden.

Maßnahmen zur Qualitätssicherung oder Verstetigung der Schulungseffekte werden ebenfalls zu selten durchgeführt. In fast zwei Drittel der Kliniken werden die Outcomes der Schulungsangebote nicht überprüft, und Qualitätszirkel bestehen sogar nur in jeder fünften Einrichtung. Da auch nur in Ausnahmefällen Auffrischungssitzungen von den Kliniken angeboten werden (können), 
scheint eine stärkere Vernetzung mit Nachsorgeeinrichtungen dringend geboten, um die Effekte der Schulungs- und Trainingsmaßnahmen dauerhaft zu sichern. In Anbetracht der internationalen Forschungsbefunde zu den stabilisierenden Effekten schriftlicher Aktionspläne für ein langfristig effizientes Selbstmanagement sollte auch diesem Aspekt künftig mehr Beachtung geschenkt werden.

Die beschriebenen Probleme in der stationären Schulungspraxis sind sicherlich in erster Linie auf schwierige institutionelle Rahmenbedingungen zurückzuführen. So ist in vielen Kliniken eine mangelnde personelle Kapazität und eine daraus resultierende Arbeitsüberlastung oder Demotivierung der Schuler zu beobachten. Vielfach müssen die Schulungsangebote als Zusatzleistungen neben den eigentlichen Aufgabenbereichen der Mitarbeiter geleistet und je nach deren aktuell verfügbaren Kapazitäten verteilt werden. Aus der mangelnden Kontinuität in der Zuständigkeit und anderen organisatorischen Gründen resultiert häufig eine hohe Fluktuationsrate unter den Schulern, die eine beständige Weiterführung der Angebote behindert.

Auch hinsichtlich der Schulerqualifikation besteht noch erheblicher Verbesserungsbedarf, da heute in der Mehrzahl Mitarbeiter ohne entsprechende Fortbildungen die Schulungen zu tragen haben. Die mittlerweile existierenden Fortbildungsangebote zum Asthma-Patientenschuler für den stationären Bereich („Trainthe-Trainer"-Seminare) sollten dementsprechend noch ausgeweitet und ortsnah durchgeführt werden, um die Qualifizierungsquote zu erhöhen. Dabei wäre mittelfristig anzustreben, auch diese Fortbildungen nach definierten Qualitätsstandards zu harmonisieren und durch die Fachgesellschaften zertifizieren zu lassen $[32,46]$. Auf der Ebene der individuellen Schuler spielen vermutlich darüber hinaus auch persönliche Faktoren (individuelle Präferenzen, eigener Stil, „eklektizistische“ Orientierung) eine entscheidende Rolle für die unzureichende Realisierung der standardisierten Schulungsmodelle. Dabei ist allerdings zu betonen, dass eine geringe Programm-Compliance der Schuler nicht mit mangelnder Schulungqualität gleichzusetzen ist. Sicherlich gibt es zahlreiche kompetente und erfahrene Schulungspraktiker, die die evaluierten Schulungsprogramme als zu statisch bzw. unflexibel ablehnen und dennoch vorbildliche Patiententrainings anbieten.

Für eine weitere Steigerung der Effizienz stationärer Asthmaschulungen scheint neben einer verstärkten Rezeption der evidenzbasierten Expertenempfehlungen vor allem eine bessere Ausstattung mit personellen und finanziellen Ressourcen sowie die Einführung von Qualitätssicherungsmaßnahmen in der Praxis erforderlich.

\section{Literatur}

${ }^{1}$ NHLBIWHO - National Heart, Lung, and Blood Institute \& World Health Organization. Global strategy for asthma mangagement and prevention. Online-document: www.ginasthma.com. 1995

2 British Thoracic Society. Guidelines on the management of asthma. Thorax 1993; 47: 1 - 24

${ }^{3}$ Wettengel R, Berdel D, Hofmann D, Krause J, Kroegel C, Kroidl RF, Leupold W, Lindemann H, Magnussen H, Meister R, Morr H, Nolte D, Rabe KF, Reinhardt D, Sauer R, Schultze-Werninghaus G, Ukena D, Worth H. Asthmatherapie bei Kindern und Erwachsenen - Empfehlungen der
Deutschen Atemwegsliga in der Deutschen Gesellschaft für Pneumologie. Med Klin 1998; 93: 639-650

${ }^{4}$ Bergmann KC, Fischer J, Schmitz M, Petermann F, Petro W. Die stationäre pneumologische Rehabilitation für Erwachsene: Zielsetzung diagnostische und therapeutische Standards - Forschungsbedarf. Pneumologie 1997; 5: 523-532

${ }^{5}$ Mühlig S, Schulz M, de Vries U, Petermann F. Grundlagen der Patientenschulung bei Asthma. In: Petermann F, Warschburger P (Hrsg.). Asthma bronchiale. Göttingen: Hogrefe, 2000: 147-174

${ }^{6}$ Petermann F. Patientenschulung und Patientenberatung - Ziele, Grundlagen und Perspektiven. 2., vollst. überarb. u. erw. Auflage. Göttingen: Hogrefe, 1997

${ }^{7}$ Schultz K, Schwiersch M, Petro W, Mühlig S, Petermann F. Individualisiertes, modular strukturiertes Patientenverhaltenstraining bei obstruktiven Atemwegserkrankungen im Rahmen der stationären Rehabilitation. Pneumologie 2000; 54: 296-305

${ }^{8}$ Arbeitsgruppe Patientenschulung der Deutschen Gesellschaft für Pneumologie und Deutsche Atemwegsliga in der Deutschen Gesellschaft für Pneumologie. Empfehlung zum strukturierten Patiententraining bei obstruktiven Atemwegserkrankungen. Pneumologie 1995; 49: 455 - 460

${ }^{9}$ Devine EC. Meta-analysis of the effects of psychoeducational care in adults with asthma. Res Nurs Health 1996; 19: 367-376

${ }^{10}$ Clark NM, Nothwehr F. Self-management of asthma by adult patients. Patient Educ Couns 1997; 32: 5-20

${ }^{11}$ Abdulwadud O, Abramson M, Forbes A, James A, Walters EH. Evaluation of a randomised controlled trial of adult asthma education in a hospital setting. Thorax 1999; 54: 493-500

12 Allen RM, Jones MP, Oldenburg B. Randomized trial of an asthma selfmanagement program for adults. Thorax 1995; 50: 731 - 738

${ }^{13}$ Bailey WC, Kohler CL, Richards JM, Windsor RA, Brooks CM, Gerald LB, Martin B, Higgins DM, Liu T. Asthma self-management. Arch Intern Med 1999; 159: $2422-2428$

${ }^{14}$ Bolton MB, Tilley BC, Kuder J, Reeves T, Schultz LR. The cost and effectiveness of an education program for adults who have asthma. J Gen Intern Med 1991; 6: 401 - 407

${ }^{15}$ Charlton I, Charlton G, Broomfield J, Mullee MA. Audit of the effect of a nurse run asthma clinic on workload and patient morbidity in a general practice. Br J Gen Pract 1991; 41: 227-233

${ }^{16}$ de Oliveira MA, Faresin SM, Bruno VF, de Bittencourt AR, Fernandes AL. Evaluation of an educational programme for socially deprived asthma patients. Eur Respir J 1999; 14: 908 - 914

17 Garrett J, Fenwick JM, Taylor G, Mitchell E, Rea H. Prospective controlled evaluation of the effect of a community based asthma education centre in a multiracial working class neighbourhood. Thorax 1994; 49: $976-983$

${ }^{18}$ Hilton S, Sibbald B, Anderson HR, Freeling P. Controlled evaluation of the effects of patient education on asthma morbidity in general practice. Lancet 1986; 1: 26-29

${ }^{19}$ Ignacio-Garcia JM, Gonzalez-Santos P. Asthma self-management education program by home monitoring of peak expiratory flow. Am J Respir Crit Care Med 1995; 151: 353 - 359

20 Jones KP, Mullee MA, Middleton M, Chapman B, Holgate ST, The British Thoracic Society Research Committee. Peak flow based asthma selfmanagement: a randomised controlled study in general practice. Tho$\operatorname{rax} 1995$; 50: 851 - 857

${ }^{21}$ Kotses H, Bernstein L, Bernstein DI, Reynolds RVC, Korbee L, Wigal JK Ganson E, Stout C, Creer TL. A self-management program for adult asthma. Part I: Development and evaluation. J Allergy Clin Immunol 1995; 95: 529-540

${ }^{22}$ Lahdensuo A, Haahtela T, Herrala J, Kava T, Kiviranta K, Kuusisto P, Perämäki E, Poussa T, Saarelainan S, Svahn T. Randomised comparison of guided self-management and traditional treatment of asthma over one year. BMJ 1996; 312: 748 - 752

${ }^{23}$ Levy ML, Robb JA, Doherty C, Bland JM, Winter RJD. A randomized controlled evaluation of specialist nurse education following accident and emergency department attendance for acute asthma. Respir Med 2000; 94: 900-908

${ }^{24}$ Mayo PH, Richman J, Harris W. Results of a program to reduce admissions for adult asthma. Ann Intern Med 1990; 112: 864-871

${ }^{25}$ Mulloy E, Donaghy D, McQuigley C, Nicholas WT. A one-year prospective audit of an asthma education programme in an out-patient setting. Ir Med J 1996; 89: 226 - 228

${ }^{26}$ Rand CS, Nides M, Cowles MK, Wise RA Connett and the Lung Health Study Group. Longterm metered-dose inhale adherence in a clinical trial. Am J Respir Crit Care Med 1995; 152: 580-588 
${ }^{27}$ Ringsberg KC, Wiklund I, Wilhelmsen L. Education of adult patients at an "asthma school": effects on quality of life, knowledge and need for nursing. Eur Respir J 1990; 3: 33-37

${ }^{28}$ Wilson SR, Scamagas P, German DF, Hughes GW, Lulla S, Coss S, Chardon L, Thomas RG, Starr-Schneidkraut N, Stancavage FB, Arsham GM. A controlled trial of two forms of self-management education for adults with asthma. Am J Med 1993; 94: 564-576

${ }^{29}$ Yoon R, McKenzie DK, Bauman A, Miles DA. Controlled trial evaluation of an asthma education programme for adults. Thorax 1993; 48: $1110-1116$

${ }^{30}$ Gibson PG, Coughlan J, Wilson AJ, Abramson M, Bauman A, Hensley MJ, Walters EH. Self-management education and regular practitioner review for adults with asthma. Cochrane Database Syst Rev 2. Oxford: Update Software , 2000

${ }^{31}$ Gibson PG, Coughlan J, Wilson AJ, Hensley MJ, Abramson M, Bauman A, Walters EH. Limited (information only) patient education programs for adults with asthma. Cochrane Database Syst Rev 2. Oxford: Update Software, 2000

${ }^{32}$ Worth H, Petro W. Vorschläge zu Struktur und Inhalten von Train-theTrainer-Seminaren für die Schulung von Patienten mit chronisch obstruktiven Atemwegserkrankungen. Pneumologie 1998; 52: 474-475

${ }^{33}$ Mühlig S. Asthmaschulung - Möglichkeiten und Grenzen des Transfers zwischen Forschung und Praxis. Prax Klin Verh Med Rehab 2001; 54: $115-119$

${ }^{34}$ Reusch A, Mühlig S. Forschungsmethodische Problemstellungen bei der Evaluation von Patientenschulungen. Prax Klin Verh Med Rehab 2000; 51: 27-30

${ }^{35}$ Reusch A, Faller H, Zwingmann C. Evaluation von Patientenschulungen: Experimentelle Designs in der Praxis. Prax Klin Verh Med Rehab 2001; 54: 107- 114

${ }^{36}$ Petro W. Patientenschulung für Atemwegskranke. München: Dustri, 1989

${ }^{37}$ Schultz K, Stark HJ, Petro W. Modulares, maßgeschneidertes Patientenverhaltenstraining. In: Petro W (Hrsg). Patientenverhaltenstrai- ning bei obstruktiven Atemwegserkrankungen München: Dustri, 1997: $142-162$

${ }^{38}$ Mühlhauser I, Richter B, Kraut D, Weske G, Worth H, Berger M. Evaluation of a structured treatment and teaching programme of asthma. J Intern Med 1991; 230: 157-164

${ }^{39}$ Münks-Lederer C, Dhein Y, Richter B, Worth H. Evaluation eines ambulanten strukturierten Asthma-Schulungsprogramms für Erwachsene. Pneumologie 2001; 55: 84-90

${ }^{40}$ Trautner C, Richter B, Berger M. Cost-effectiveness of a structured treatment and teaching programme on asthma. Eur Respir J 1993; 6 : $1485-1491$

${ }^{41}$ Wöller W, Kruse J, Alberti L, Kraut D, Richter B, Worth H, Tress W. Affektiv-kognitive Anfallsverarbeitung und Krankheitsverhalten bei Patienten mit Asthma bronchiale. Psychother Psychosom Med Psychol 1992; 42: $63-70$

42 Worth H. Zum Stellenwert der Patientenschulung in der Therapie des Asthma bronchiale. Prax Klin Verh Med Rehab 1989; 6: 84-87

${ }^{43}$ Barczok M. Patientenschulung aus der Sicht des Berufsverbandes der Pneumologen. In: Petro W (Hrsg). Patientenverhaltenstraining bei obstruktiven Atemwegserkrankungen. München-Deisenhofen: Dustri, 1997: 1 - 7

44 Deutsche Gesellschaft für Pneumologie/Deutsche Atemwegsliga. Wer bietet was? Information, Schulung, Verhaltenstraining - Einrichtungen für Patienten mit obstruktiven Atemwegserkrankungen. München: KLINGE PHARMA, 2000

${ }^{45}$ Petro W. Patientenverhaltenstraining bei obstruktiven Atemwegserkrankungen - Analyse der Quantität und Qualität. In: Petro W (Hrsg). Patientenverhaltenstraining bei obstruktiven Atemwegserkrankungen. München: Dustri, 1997: 176-188

${ }^{46}$ Worth H. Train-the-Trainer-Seminare (TTS) für die Patientenschulung erwachsener Asthmatiker-Strategien und erste Erfahrungen. In: Petro W (Hrsg). Patientenverhaltenstraining bei obstruktiven Atemwegserkrankungen. München: Dustri, 1997: 75-82 